\title{
Neonatal HIV-associated nephropathy
}

R Bhimma, ${ }^{1}$ MB ChB, DCH, FCP (Paeds), MMed, MD, Cert Nephrol (Paeds);

E Naicker, ${ }^{1} \mathrm{MB}$ ChB, DCH, FCP (Paeds), Cert Nephrol (Paeds); B W Mzimela, ${ }^{2} \mathrm{MB}$ ChB

${ }^{1}$ Department of Paediatrics and Child Health, School of Clinical Medicine, College of Health Science, Nelson R Mandela School of Medicine, University of KwaZulu-Natal, Durban, South Africa

${ }^{2}$ Department of Paediatrics and Child Health, Inkosi Albert Luthuli Central Hospital, Durban, KwaZulu-Natal, South Africa

Corresponding author: RBhimma (bhimma@ukzn.ac.za)

\begin{abstract}
Human immunodeficiency virus (HIV)-related nephropathy is a significant cause of morbidity and mortality in HIV-1 seropositive children in Africa that presents at any age. To date the youngest patient reported in the literature was from our centre, presenting at 4 months of age. We present here a neonate born to an HIV-1 infected mother on combined anti-retroviral therapy (cART), who had vertical transmission of the virus and presented with congenital nephrotic syndrome at three weeks of life. The child was confirmed to have HIV-1 infection at 6 weeks. Kidney biopsy showed features consistent with HIV-associated nephropathy. On commencement of cART and angiotensin converting enzyme antagonist treatment, there was a substantial decrease in proteinuria. To the best of our knowledge, this is the first report of HIV-associated nephropathy presenting as congenital nephrotic syndrome.
\end{abstract}

S Afr J Child Health 2018;12(1):38-40. DOI:10.7196/SAJCH.2018.v12i1.1437

The earliest reports of childhood HIV-1-associated nephropathy (HIVAN) were in African-American children from Miami and New York in $1984 .{ }^{[1]}$ Subsequently, several forms of kidney disease in HIV-1-endemic regions have been described in both children and adults, ${ }^{[2]}$ resulting from the direct effects of HIV-1 on the kidney or indirect effects of intercurrent illness or medications. HIV-related nephropathy is now recognised as a significant cause of morbidity and mortality in HIV-1-seropositive children in Africa. ${ }^{[3]}$ It is among the ten most common non-infectious conditions occurring in perinatal HIV-infected children and adolescents in the highly active anti-retroviral therapy era. ${ }^{[4]}$

Based on currently available literature, the youngest age of presentation of a child with HIVAN was 4 months in a series published from our centre in Durban, KwaZulu-Natal, South Africa (SA). ${ }^{[3]}$ HIVAN is almost exclusively found in black patients, with African-Americans having a fourfold increased risk of progression to end-stage kidney disease. ${ }^{[5]}$ HIVAN was initially reported as being a late presentation of HIV infection. ${ }^{[6]}$ Subsequently, following the recognition of the disease in children, HIVAN was identified in younger children around 2 to 3 years of age. ${ }^{[1]}$ In one of the largest reported series of children with HIV-related kidney disease in Africa, 19 children (37.3\%) were younger than 5 years; the youngest patient was 4 months old with features of HIVAN on histopathology. ${ }^{[3]}$ In another series of 10 Nigerian patients, the youngest was aged 5 months. ${ }^{[7]}$

To date, there have been no reports in the literature of HIVAN presenting with congenital nephrotic syndrome (CNS). We report here what we believe is the first case of a child with CNS, who was diagnosed with perinatally acquired HIV-1 infection due to vertical transmission.

\section{Case report}

A 7-week-old female child born to non-consanguinous Mozambican parents presented at 3 weeks of age with poor appetite, skin lesions, anarsaca, severe hypoalbuminaemia $(8.0 \mathrm{~g} / \mathrm{L})$, and anaemia (haemoglobin $9.2 \mathrm{~g} / \mathrm{dL}$ ). The child was born at term by normal vaginal delivery with a birth weight of $3.2 \mathrm{~kg}$ and Apgar scores of 8/10 and $9 / 10$ at 5 and 10 minutes, respectively. The placental weight was not available. The mother had been booked at 24 weeks gestation and tested HIV-positive with a $\mathrm{CD} 4^{+}$cell count of 98 cells/ $\mu \mathrm{L}$. Unfortunately, her HIV viral load was not available. She was initiated on fixed-dose combined antiretroviral therapy (cART) using ATRIPLA (efavirenz, emtricitabine (FTC) and tenofovir disoproxil fumerate) and the child was initiated on the elimination of mother-to-child transmission (EMTCT) programme using nevirapine ( $2 \mathrm{mg} / \mathrm{kg}$ for 6 weeks). The child was diagnosed with severe acute malnutrition and managed in accordance with World Health Organization guidelines. After a week, the oedema failed to resolve and she was initiated on daily albumin infusions for 6 days. Urinary dipstick analysis that was initially negative showed proteinuria of $3+$ following albumin infusions and the urinary protein $(\mathrm{mg} / \mathrm{dL})$ :creatinine $(\mathrm{mg} / \mathrm{dL}$ ) ratio was 3.0 (normal ratio $<0.5$ ), which was consistent with massive proteinuria. The child was subsequently referred to Inkosi Albert Luthuli Central Hospital (IALCH) in Durban for further management.

At IALCH the child was oedematous, haemodynamically stable, with a normal blood pressure of $90 / 46 \mathrm{mmHg}$ ). Her urinary protein:creatinine ratio on admission was 4.2. A 24-hour urine sample confirmed massive proteinuria of $5.04 \mathrm{~g} / 24$ hours (normal value $\leq 29 \mathrm{mg} / 24$ hours). The serum albumin was $23 \mathrm{~g} / \mathrm{L}$ and serum cholesterol was $4.5 \mathrm{mmol} / \mathrm{L}$. The child was hyponatraemic with a serum sodium of $125 \mathrm{mmol} / \mathrm{L}$ and mildly acidotic (serum bicarbonate $18 \mathrm{mmol} / \mathrm{L}$ ) with a disproportionately raised blood urea and low serum creatinine, which was suggestive of pre-renal failure. Serological tests were conducted for toxoplasmosis, EpsteinBarr virus, hepatitis $B$ and $C$, syphilis (rapid plasma reaction) and CMV (including a qualitative polymerase chain reaction (PCR) test). In addition, staining for Mycobacterium tuberculosis was conducted on a renal biopsy. Serum complement, lipid levels and cardiac echocardiography were normal. Unfortunately, genetic testing for the various candidate genes implicated in CNS was not undertaken as this was unavailable for routine clinical care. The mother tested negative for hepatitis B and C, syphilis, tuberculosis and lupus, with evidence of past exposure to cytomegalovirus. Ultrasound of the child's kidneys showed both kidneys to be normal in position and size but echogenic with poor cortico-medullary differentiation with the right and left kidney measuring $5.6 \mathrm{~cm}$ and $5.5 \mathrm{~cm}$, respectively. There was no evidence of hydronephrosis, hydroureter or perinephric collections. The child was commenced on an angiotensin converting enzyme antagonist (enalapril), thiazide diuretic (hydrochlorothiazide) and an aldosterone antagonist (spironolactone), with ongoing cART. There was no evidence of extra- 
renal manifestations of CNS such as neurological, ophthalmological, haematological, cutaneous and pulmonary manifestations. At 6 weeks, the child's HIV test using reverse transcriptase anti-DNA PCR (HIV Viral Load Kit, Roche Diagnostics, SA) was positive and she was initiated on cART. An open kidney biopsy by the surgical team showed 38 glomeruli on light microscopy, with no global sclerosis. Several glomeruli showed focal and segmental increase in mesangial cellularity and sclerosis. There was no evidence of periglomerular fibrosis, crescent formation or karyorrhexis. Spike formation and double contours were not seen on special stains. The adjacent tubulointerstitium showed minimal fibrosis but no interstitial inflammation. Tubules did not show microsystic tubular dilatation and there were no established alternations of benign hypertension or accelerated nephroangiosclerosis. Infective pathogens were not seen. The kidney biopsy specimen for immunofluorescence unfortunately showed only one glomerulus that stained negative for $\mathrm{C} 1$. Staining for C3, C4, IgA, IgG and IgM was negative. Electron microscopy showed subepithelial deposits and variable thickening of the glomerular basement membrane. There was podocyte foot process effacement and minimal mesangial sclerosis. Viral inclusions were not seen. Despite the poor representation of glomeruli on the specimen used for immunofluorescence, the overall features on histopathology favoured a histopathological diagnosis of HIV-immune complex kidney disease. Unfortunately, immunohistochemistry was not done.

After two doses of albumin infusions, the child's oedema resolved completely and no further albumin infusions were necessary. Her urinary protein:creatinine ratio decreased from 4.2 to 2.6 and her discharge albumin was $23 \mathrm{~g} / \mathrm{dL}$, despite not having had albumin infusions for a fortnight (Table 1). The child was discharged and unfortunately lost to follow-up.

\section{Discussion}

CNS is a rare but severe form of nephrotic syndrome presenting in the first three months of life. The disease is characterised by heavy proteinuria, hypoproteinaemia and oedema, which most often progresses to anasarca. The degree of proteinuria immediately after birth is variable and hence clinical signs of nephrotic syndrome may only present after a few weeks of life, with the true magnitude of proteinuria sometimes only becoming apparent after partial correction of hypoalbuminaemia following albumin infusions. ${ }^{[8]}$ The disease is either primary or secondary to systemic diseases, typically perinatal infections, including congenital syphilis, rubella, toxoplasmosis, cytomegalovirus and hepatitis $B .{ }^{[9]}$ Non-infectious cases of CNS have been reported in association with maternal systemic lupus erythematosus and neonatal alloimmunisation against neonatal endopeptidase present on podocytes. ${ }^{[10]}$ The primary or genetically inherited forms are most commonly associated with mutations encoding structural proteins of the slit diaphragm, i.e. nephrin (NPHS1) and podocin (NPHS2), as well as a transcription factor (WT1).
HIV-1 has been implicated as a secondary cause of CNS; $;{ }^{[9]}$ however, an extensive literature search failed to reveal any report of CNS secondary to HIV infection. The youngest child reported to date has been a 4-month-old from our centre. ${ }^{[3]}$ To the best of our knowledge, this is the first case of CNS reported from and HIV-1 endemic area. The spectrum of kidney disease described to date that occurs with perinatal HIV-1 infection in children encompasses chronic glomerular disorders, such as HIVAN and HIV immune complex kidney disease, the thrombotic microangiopathies (including atypical forms of haemolytic uraemic syndrome and thrombotic thrombocytopenic purpura), disorders of proximal tubular function and acute kidney injury. ${ }^{[4]}$ All of these disorders have been reported in older patients, of which the youngest was 4 months old. This is older than the patient we presented in this report who was only 7 weeks old. Also, HIVAN or HIV-immune complex kidney disease has not been reported as part of the spectrum of CNS.

Although the mother had been commenced on cART early in her pregnancy, she had a low CD4 count with a high viral load, making a strong case for perinatal transmission of the virus. The child tested positive at 6 weeks of age using HIV-1 polymerase chain reaction thus confirming infection. The classical presentation of CNS in the setting of HIV-1 infection with a biopsy finding consistent with HIV immune complex disorder will strongly support HIV-1 as a secondary cause of CNS in this case, although tissue for immunofluorescence was suboptimal and lights microscopy did not show microcytic tubular dilatation (a typical finding of nephropathy in the setting of HIV). Electron microscopy however strongly supported a diagnosis of HIV-1 immune complex kidney disease showing subepithelial deposits and variable thickening of the glomerular basement membrane. Failure to demonstrate other common known pathogens causing CNS further supports a diagnosis of HIV-1 associated immune complex disease. HIVAN is a distinct entity from immune complex glomerulopathy and membranous glomerulopathy. It can be difficult to differentiate immune complex glomerulpathy (HIVICK) from membranous glomerulopathy in a patient with HIV because HIVICK has varying morphologies, including a membranous pattern. Thus, a secondary membranous glomerulopathy and HIVICK will have the same histomorphology ${ }^{[11]}$

It is possible that the findings of HIV-1 infection in the case of a child with CNS in a highly endemic region may be purely coincidental. However, to date, primary CNS (genetic form) has not been reported with histopathological findings of immune complex kidney disease. Genetic forms of CNS may cause histopathological lesions, including mesangial expansion, minimal change disease, focal segmental glomerulosclerosis, and diffuse mesangial sclerosis (with or without tubular dilatation, interstitial fibrosis and inflammation). ${ }^{[12]}$ However, to date, primary CNS (genetic form) has not been reported with histopathological findings of immune complex kidney disease.

The response to cART and angiotensin converting enzyme therapy,

Table 1. Laboratory findings during admission to Inkosi Albert Luthuli Central Hospital

\begin{tabular}{lllllll}
\hline & \multicolumn{5}{c}{ Date } \\
\cline { 2 - 7 } Sodium $(\mathrm{mmol} / \mathrm{L})$ & $\mathbf{0 3 / 0 1 / 1 7}$ & $\mathbf{0 4 / 0 1 / 1 7}$ & $\mathbf{0 6 / 0 1 / 2 0 1 7}$ & $\mathbf{1 6 / 0 1 / 2 0 1 7}$ & $\mathbf{1 9 / 0 1 / 2 0 1 7}$ & $\mathbf{2 3 / 0 1 / 2 0 1 7}$ \\
\cline { 2 - 7 } & 125 & 132 & 131 & 124 & 129 & 138 \\
Potassium $(\mathrm{mmol} / \mathrm{L})$ & 5.5 & 5.4 & 4.9 & 5.8 & 5.9 & 4.9 \\
Chloride $(\mathrm{mmol} / \mathrm{L})$ & 97 & 101 & 107 & 97 & 104 & 108 \\
Bicarbonate $(\mathrm{mmol} / \mathrm{L})$ & 18 & 13 & 12 & 22 & 20 & 27 \\
Urea $(\mathrm{mmol} / \mathrm{L})$ & 11.2 & 9.9 & 7.6 & 5.7 & 3.8 & 2.5 \\
Creatinine $(\mu \mathrm{mol} / \mathrm{L})$ & 15 & $<9$ & $<9$ & $<9$ & $<9$ & $<9$ \\
Albumin $(\mathrm{g} / \mathrm{L})$ & 23 & 17 & 19 & 13 & 15 & 23 \\
Urine protein $(\mathrm{mg} / \mathrm{dL}):$ Creatinine $(\mathrm{mg} / \mathrm{dL})$ & & & 4.2 & & & 2.6
\end{tabular}


with partial remission of the disease without the need for constant albumin infusions, favours a secondary form of CNS, as the primary (genetic) forms are usually recalcitrant to therapy. The control of oedema in these children requires multiple therapeutic interventions. Unfortunately, the child was lost to follow-up and her response to cART and angiotensin inhibitor therapy could not be assessed.

\section{Conclusion}

We believe this is the first case of neonatal HIVAN reported in the literature that has been confirmed on histology. The long-term response to cART and angiotensin inhibitor therapy could not be assessed due to loss to follow-up.

Acknowledgements. We would like to thank the medical manager of Inkosi Albert Luthuli Hospital for permission to publish and Miss Louansha Nandlal for her assistance in drafting the manuscript.

Author contributions. All authors were involved in the clinical care of the patient. E Naicker and BW Mzimela were involved with the writing of the clinical case and review of the final draft of the paper.

Funding. None.

Conflicts of interest. None.

1. Pardo V, Meneses R, Ossa L, et al. AIDS-related glomerulopathy: Occurrence in specific risk groups. Kidney Int 1987;31(5):1167-1173.

2. Nochy D, Glotz D, Dosquet P, et al. Renal disease associated with HIV infection: A multicentric study of 60 patients from Paris hospitals. Nephrol Dial
Transplant 1993;8(1):11-19.

3. Ramsuran D. The Spectrum of HIV Related Nephropathy in KwaZuluNatal: A Pathogenetic Appraisal and Impact of HAARTT. Durban: University of Kwazulu-Natal, 2012. https://researchspace.ukzn.ac.za/xmlui/ handle/10413/10447 (accessed 12 November 2017)

4. Bhimma R, Udharam M, Kala U. Kidney disease in children and adolescents with perinatal HIV-1 infection. J Int AIDS Soc 2013;16(1). https://doi. org/10.7448/IAS.16.1.18596

5. Kopp JB, Nelson GW, Sampath K, et al. APOL1 Genetic variants in focal segmental glomerulosclerosis and HIV-associated nephropathy. J Am Soc Nephrol 2011;22(11):2129-2137. https://doi.org/10.1681/ASN.2011040388

6. Brasile L, Green E, Haisch C. Ex vivo resuscitation of kidneys after postmortem warm ischemia. ASAIO J 1997;43(5):427-430.

7. Anochie IC, Eke FU, Okpere AN. Human immunodeficiency virus-associated nephropathy (HIVAN) in Nigerian children. Pediatric Nephrol 2008;23(1):117122. https://doi.org/10.1007/s00467-007-0621-0

8. Jalanko H. Congenital nephrotic syndrome. Pediatric Nephrol 2009;24(11):2121-2128.

9. Barratt TM, Avner ED, Harmon WE. Pediatric Nephrology, 4th ed. Baltimore: Lippincott, Williams and Wilkins, 1999:933-945.

10. Kerjaschki D. Pathomechanisms and molecular basis of membranous glomerulopathy. Lancet 2004;364(9441):1194-1196. https://doi.org/10.1016 S0140-6736(04)17154-7

11. Motala AI, Mogotlane L, Goetsch S. Renal pathology in the HIV-positive child: From nephrosis to nephritis and everything in between. Diagnostic Histopathol 2009;15(5):232-240. https://doi.org/10.1016/j.mpdhp.2009.02.012

12. Jalanko H, Holmberg C. Congenital nephrotic syndrome. Pediatr Nephrol 2009; 24(11): 2121-2128. https://doi.org/10.1007\%2Fs00467-007-0633-9

Accepted 28 September 2017. 